\title{
Patello-femoral Replacement
}

\author{
Romagnoli Sergio, Petrillo Stefano, \\ and Marullo Matteo
}

\section{Key Points}

- Clinical results and survivorship of PFA are improving thanks to a better understanding of the PF biomechanics, more anatomical designs and adequate surgical instrumentation.

- Onlay designs have broader indications and easier surgical technique because they completely replace the trochlea by an anterior resection. Their patella-friendly trochlea and the large amount of sizes available accommodate for patella alta or excessive TT-TG distance without the need of further surgical procedures.

- PFA in PFOA secondary to patellar instability needs a kinematic alignment in which the lateral border of the trochlea is elevated, the lateral trochlear inclination is restored, but the trochlear line remains in partial external rotation. This avoids excessive modification in retinacular ligaments tension and the need of soft tissues releases.

- PFA in primary PFOA without any trochlear dysplasia can be performed with an anatomical alignment. The anterior cut should be perpendicular to the sagittal axis of the kneevjoint and the prosthesis should replace the trochlea without modifying its anatomy and orientation. Inlay PFAs could be used.

R. Sergio $(\bowtie) \cdot$ P. Stefano $\cdot$ M. Matteo Joint Replacement Department, IRCCS Galeazzi Orthopaedics Institute, Milan, Italy e-mail: Sergio.romagnoli@libero.it; s.petrillo@unicampus.it

(C) The Author(s) 2020

C. Rivière, P.-A. Vendittoli (eds.), Personalized Hip and Knee Joint Replacement, https://doi.org/10.1007/978-3-030-24243-5_20

\subsection{Introduction}

The first isolated patello-femoral (PF) joint arthroplasty (PFA) was a patella cap, a Vitallium shell replacing the patella and maintaining the native trochlea, proposed in 1955 by McKeever. The first PFAs replacing entire PF joint had an inlay design and came in the 1979 with the Richards and Lubinus prosthesis.

I started performing PFA in the first 1980s using inlay PFAs, the cementless Bousquet and the Cemented Cartier prosthesis. Then I have continued in the 1990s with the half-inlay Grammont and the inlay Lubinus prosthesis. However, firstgeneration PFAs had important limitations and poor outcomes. These PFAs were inserted in the native trochlea, replacing the articular cartilage and leaving untouched the subchondral bone, without correcting the rotational alignment of the trochlea. These unideal designs with few component sizes available, improper surgical technique, inadequate instrumentation and unfavourable indications provided poor outcomes. Indeed, good or excellent results were achieved in only $20-72 \%$ of patients at short-term and midterm follow-up, with a high incidence of early reoperations (25-35\% at 5 years) due to patellar maltracking, instability, patellar clunking and soft tissue impingement [1].

In the last 20 years, I preferred the onlay PFAs, using the inlay designs only in few selected cases. Onlay prostheses completely resect the 
trochlea with an anterior cut similar to the one performed for total knee arthroplasty (TKA). The Avon (Stryker) and the Zimmer PFJ are examples of onlay prostheses. Second-generation PFAs allow a correction of trochlea rotation or dysplasia and are associated with good results at shortterm and midterm follow-up [1]. The enhanced knowledge on PF kinematics, the higher number of component sizes available, the better surgical instrumentation and the easier surgical technique contributed to improve the results. Moreover, early complications like patellar maltracking, instability or catching and snapping of the patellar component during knee flexion were significantly reduced.

\subsection{Biomechanics of the PF Joint}

PF joint (PFJ), among all the knee compartments, has the most complex biomechanics. An extensive knowledge about forces acting on the PFJ as well as variables affecting them is crucial to understand the surgical technique of PFA.

Forces on the PFJ act in the sagittal, coronal and axial plane. In the sagittal plane, there is a parallelogram of forces created by the quadriceps strain force $(\mathrm{QF})$ and the patellar tendon strain force (PTF). The resultant vector between these two forces is defined as the PF reaction force (PRF). It is the "pressure" on cartilage of the patella and of the trochlea. PRF increases with flexion and decreases with the anterior displacement of the tibial tuberosity (Fig. 20.1) [2].

The moment arm acting on the PFJ depends on the distance between the vertical line created by the gravity centre and the PFJ. During stair ascending, the gravity centre comes closer to the $\mathrm{PFJ}$, and the moment arm is shorter, while during stair descending, the body weight moves behind, so the distance between the gravity centre and the PFJ increases, increasing also the loadings on all knee compartments [3].

In the coronal plane, the quadriceps strain force and the patellar tendon force draw an angle, which is generally called Q angle. The Q angle determines a lateral force vector (patellar lateral force, PLF), which is maximal in complete knee

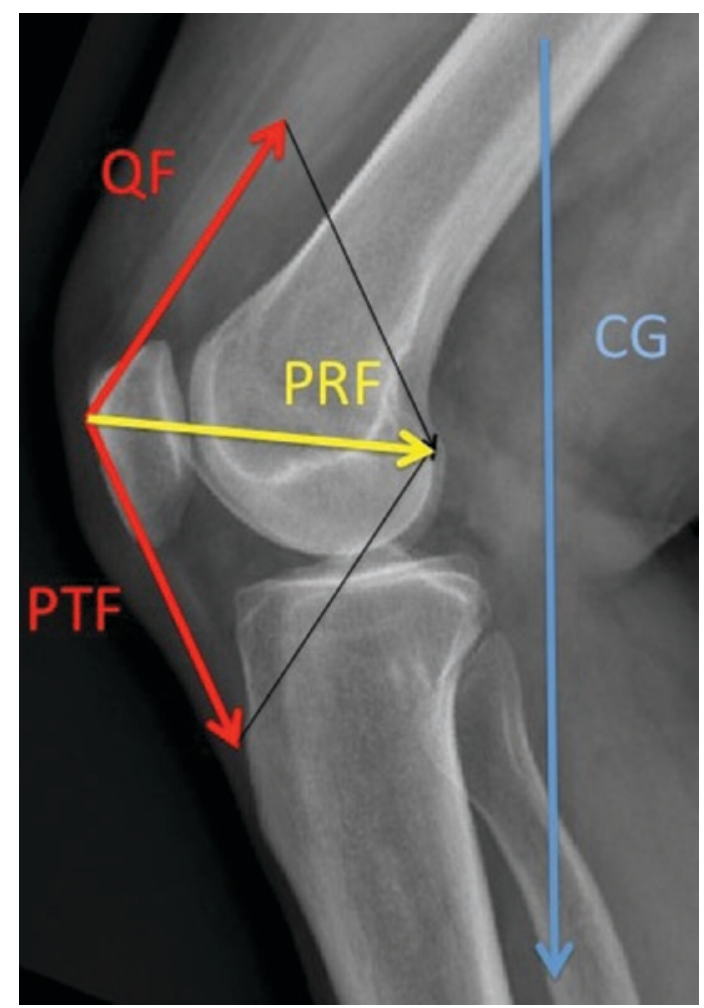

Fig. 20.1 Forces acting on the patella in the sagittal plane. The resultant vector between the quadriceps strain force $(\mathrm{QF})$ and the patellar tendon strain force (PTF) is the patello-femoral reaction force (PRF). $C G$ gravity centre

extension. This is a factor contributing to lateral patellar dislocation, which is more frequent in complete knee extension (Fig. 20.2). During knee flexion, the internal rotation of the tibia neutralizes the $\mathrm{Q}$ angle and reduces PLF. In the coronal and axial plane, the PLF is antagonized by the reaction caused by the inclination of the lateral facet of the femoral trochlea. At $60^{\circ}$ of knee flexion, there is a condition for patellar stability, in which the lateral trochlear inclination angle is bigger than the Q angle [4].

Even in the axial plane, there is a parallelogram of forces, in which the direction and entity of the vectors depend on knee flexion, trochlear anatomy, patellar anatomy, balance of the patello-femoral ligaments and tension/action of the quadriceps muscle.

Moreover, the mechanical axis of the lower limb influences PF biomechanics. In particular, the valgus morphotype is detrimental for 


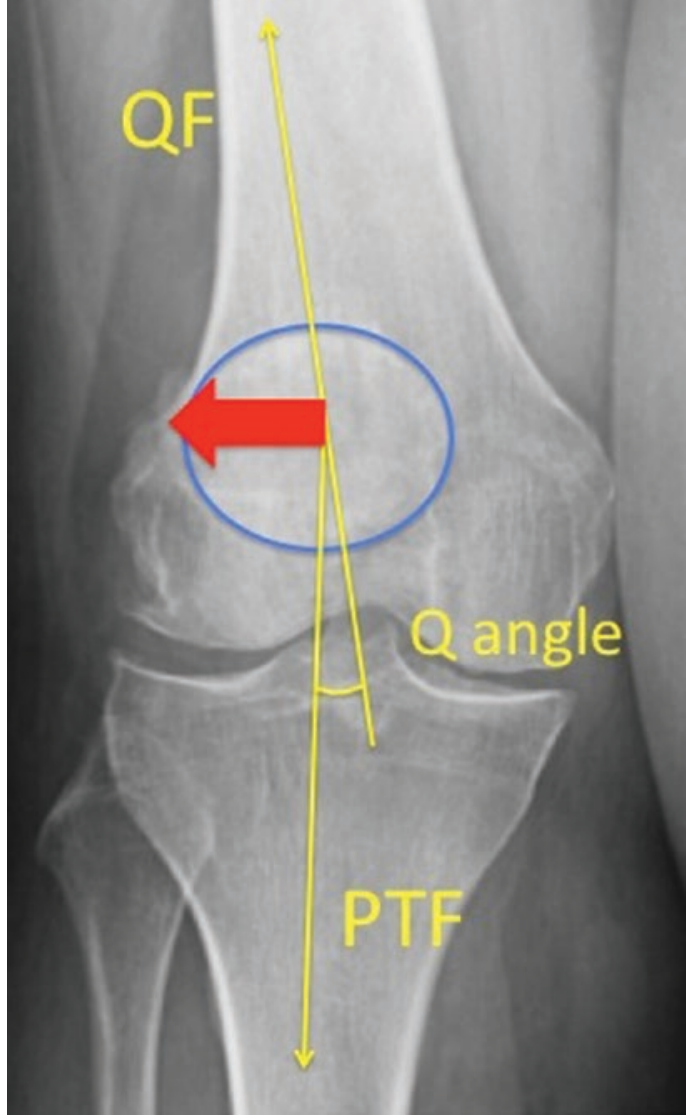

Fig. 20.2 In the coronal plane, the quadriceps strain force $(\mathrm{QF})$ and the patellar tendon force (PTF) draw the Q angle. The bigger the angle, the bigger the patellar lateral force (red arrow)

a proper PF tracking; it increases the obliquity of the $\mathrm{QF}$, thus increasing the $\mathrm{Q}$ angle and the PLF. Consequently, in high-grade valgus deformity, the patella tends to partially or completely dislocate laterally.

This condition should be considered in patients with isolated PF osteoarthritis (PFOA) and valgus deformity greater than $5^{\circ}$. In selected cases, it is possible to correct the coronal alignment of the knee by a femoral osteotomy (if the lateral tibiofemoral compartment is pristine) or a lateral unicompartmental knee arthroplasty (if the lateral tibiofemoral compartment is damaged, even if fairly symptomatic) and then add a PFA with easier alignment [5]. This shrewdness significantly reduces the risk of patellar dislocation and PFA failure (Fig. 20.3).
Gender has an important influence on PFJ biomechanics. It was shown that the mean $\mathrm{Q}$ Angle is $17^{\circ}$ in female and $14^{\circ}$ in male [6]. Moreover, females have a higher internal rotation of the femoral trochlea (trochlear angle) which is $2^{\circ}$ greater in female, mainly due to a shorter medial condyle in the sagittal plane [7]. The trochlear obliquity in the coronal plane is higher in female than in male $\left(10^{\circ}\right.$ vs. $\left.7^{\circ}\right)$ [8], while patellar thickness is higher in male than in female $(2.57 \mathrm{~cm}$ vs. $2.25 \mathrm{~cm}$, mean values). Moreover, males have higher medial and lateral trochlear facets and a wider trochlea. Consequently, females have smaller PFJ surface leading to load concentration, with also a higher Q angle and higher femoral internal rotation leading to patellar lateralization. Furthermore, the higher rates of trochlear dysplasia and ligament laxity in females facilitate malalignment, and all these factors explain why PFOA is significantly more frequent in female than males.

\subsection{Indications}

Symptomatic osteoarthritis affects the PF joint less frequently than the other compartments of the knee. Isolated PFOA has been reported in $8 \%$ of women and $2 \%$ of men over 55 years of age [9].

Three main causes determine isolated PFOA:

- Primary OA: patients with no orthopaedic antecedent and no history of patella instability. These patients are generally over 60 years old, are overweight and have a symmetrical $\mathrm{OA}$ of the medial and lateral patellar and trochlear facets.

- PF instability: patients with a history of objective patellar dislocation. These patients have evidence of trochlear dysplasia and/or patella alta. They are generally younger (mean age 54 years old) and often have a bilateral disease.

- Post-traumatic: patients with a history of PF fractures (young patients with a mean age at surgery of 54 years). 


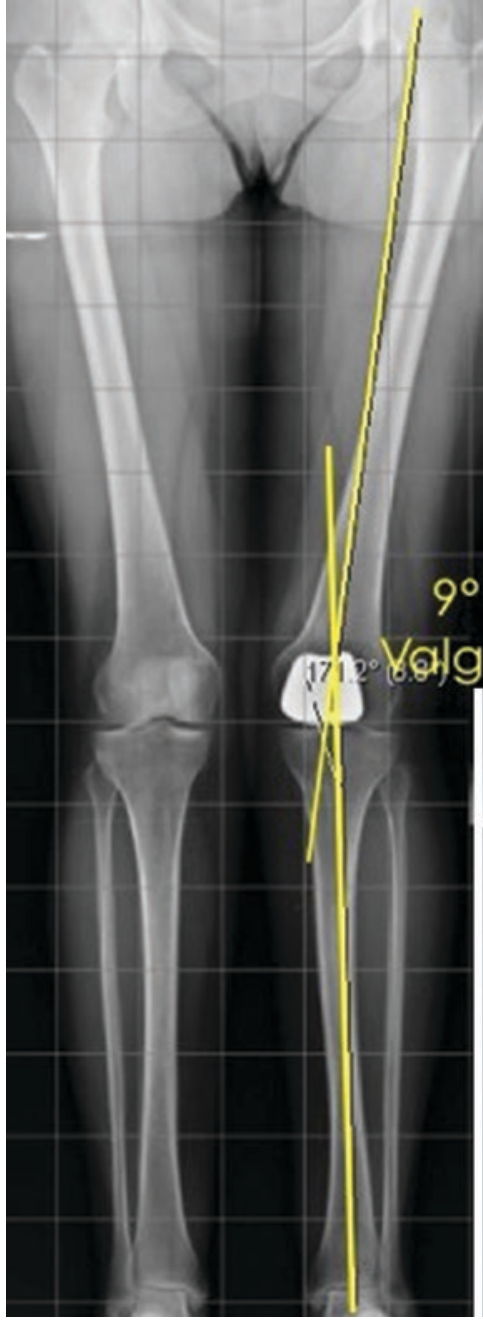

Fig. 20.3 Even if the PFA adequately replaced the native PFJ, the residual deformity ( $9^{\circ}$ valgus) in the coronal plane maintained a high $\mathrm{Q}$ angle and consequently an excessive patellar lateral force. Clinical consequences
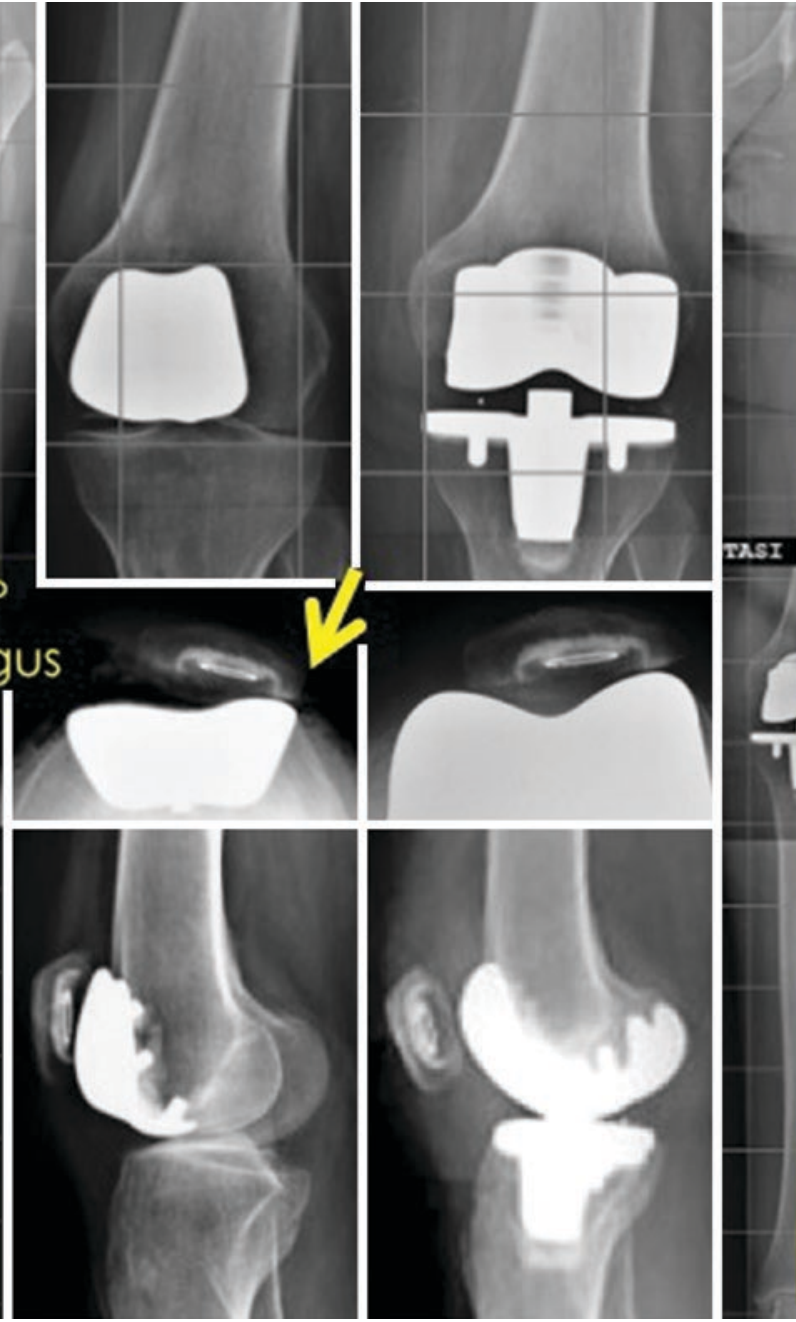

were patellar instability and partial dislocation. The correction of mechanical axis with a TKA restored a proper PF tracking
Primary OA represents $49 \%$ of cases, while post-instability $\mathrm{OA}$ and post-traumatic OA account for $33 \%$ and $9 \%$ of cases, respectively $[10,11]$. Trochlear dysplasia is the main factor leading to isolated PFOA. Indeed, $78 \%$ of all patients present trochlear dysplasia with crossing sign. The highest rate of dysplasia was seen in the post-instability group (66\%), but even the primary OA group demonstrated a 38\% of trochlear dysplasia [10, 11]. Isolated PFOA affects pre- dominantly females $(72 \%)$, with $51 \%$ of patients showing bilateral disease $[10,11]$.

Patients with isolated PFOA typically have significant anterior knee pain that interferes with several activities of daily living. They have difficulties in using stairs (often they need a handrail) and in getting up from a chair. They have also a limited walking capacity on flat surfaces. At clinical examination, the knee is often swollen, and typical retropatellar palpation or patel- 
lar compression evokes pain. Squatting for these patients is often impossible.

A proper radiographic evaluation of PFOA should include antero-posterior weight-bearing view, Rosenberg's view, lateral weight-bearing view and skyline view at $30^{\circ}$ of knee flexion (Merchant's view). It is sometimes useful to take skyline views in various degrees of flexion because the pathology may be much more obvious in one position than another. Magnetic resonance imaging can be useful to assess doubtful cases or associated soft tissues lesions.

PFA preserves both cruciate ligaments and the tibiofemoral compartments, enhances stability and maintains proprioception and a more physiological tibiofemoral kinematics compared to TKA. Clinical consequences are greater comfort during daily life activities and better functional outcomes. Moreover, PFA saves more bone stock compared to a TKA, making an eventual prosthetic revision less difficult.

Indications for isolated PFA are symptomatic isolated PFOA (Iwano grade 2 or greater) and absence of tibiofemoral arthritis (KellgrenLawrence 2 or lower).

Contraindications are a clinically instable knee in the frontal or sagittal plane, a preoperative range of motion (ROM) less than $90^{\circ}$, a flexion contracture greater than $10^{\circ}$ and inflammatory disease. The tibiofemoral cartilages should be pristine; in case one of the tibiofemoral compartments is damaged, a bicompartmental replacement (unicompartmental knee replacement, UKA and PFA) should be considered if there are no other contraindications [12].

We developed an algorithm to consider UKA in association to a PFA. There are two major criteria and two minor criteria. The two main criteria are valgus deformity greater than $5^{\circ}$ or varus deformity greater than $4^{\circ}$, high adduction moment. The two secondary criteria are female sex and body mass index (BMI) $>32$. If two major criteria or one major + two minor criteria are present, we suggest to perform a UKA + PFA. At the same time, we developed another algorithm to recognize when performing PFA in association to UKA. Three major criteria and two minor criteria compose this algorithm. The major criteria are patello-femoral pain; patellar malalignment or lateral patello-femoral wear on X-ray axial view; and intraoperative findings of grade 3-4 patello-femoral chondral degeneration. The two minor criteria are the same of the first algorithm. If two major criteria or one major + two minor criteria are present, we suggest to add a PFA to a UKA due to high risk of PFOA progression [13].

\subsection{Inlay and Onlay Designs}

PFAs could be divided in two main categories: "inlay" and "onlay" prostheses. Inlay prosthesis lies inside the native trochlea, without modifying its anatomy. These are contraindicated in PFOA with high-grade trochlear dysplasia. In patella alta or in case of excessive tibial tuberositytrochlear groove (TT-TG) distance, these inlay prostheses should be associated with other surgical procedures such as tibial tuberosity distalization or medialization.

Onlay PFA completely resects the trochlea with an anterior cut similar to the one performed for TKA. These prostheses have a bigger trochlear component compared to the inlay designs, which replaces the entire trochlea. They can be even utilized in case of high-grade trochlear dysplasia, in which the native anatomy of the trochlea is pathological and cannot be preserved, in patella alta and in cases of excessive TT-TG distance. The trochlear flange extends proximally and has a better congruency with the patella, even in case of patella alta. Increase in proximal femoropatellar contact area is of paramount importance for a proper patellar tracking in the first $30^{\circ}$ of knee flexion, the critical range in which patellar dislocation occurs. The complete resection of the native trochlea lets to modify the position of the trochlear groove to adapt it to a lateralized tibial tuberosity, decreasing the TT-TG distance by a proximal realignment.

Onlay PFAs have broader indications without necessity of associated surgical procedures, presenting an easier surgical technique and consequently a shorter learning curve. Moreover, literature showed superior results and survivorship with onlay PFAs compared to inlay ones [13-16]. 


\subsection{Surgical Technique}

The patient is supine on the operating table. We do not use the tourniquet in any type of knee replacement for several reasons (higher risk of thromboembolic disease, slower recovery, lower range of motion, higher risk of wound complications) but principally because the tourniquet does not allow the evaluation of the balance and motion of the extensor apparatus.

PFA could be performed through any standard knee replacement incision; anyway, the most utilized surgical approach is the medial parapatellar one. Our preferred approach is mini-mid vastus. The incision should be $6-8 \mathrm{~cm}$ long and correspond to the proximal part of the one used for a TKA. Care must be taken to avoid damage of the meniscus or the cartilage of the tibiofemoral joint.

A lateral approach should be considered when the patella is strongly subluxated laterally or when it is planned to perform a lateral UKA. This access gives slightly less exposure of the joint, but it does not violate the quadriceps at all. Moreover, it lets to perform a fine modulation of the lateral PF and patellotibial ligaments during capsular suture after implantation.

A careful inspection of the whole joint is suggested to confirm intraoperatively the indication for PFA or to move to another surgical option.
The trochlear cut is the first bone cut to be performed, with the knee at $90^{\circ}$ of flexion. As mentioned above, we suggest using onlay PFAs, so the trochlea should be completely resected and replaced.

In patients with primary PFOA without trochlear dysplasia, the trochlear line (TL), defined as the line connecting the anterior points of medial and lateral facets, is internally rotated relative to the posterior condylar line as the lateral facet is more prominent than the medial one and the lateral trochlear inclination (LTI) is pronounced, and the PF ligaments are balanced [17]. The PFJ works with proper biomechanics, so the PFA should not modify the native anatomy. Consequently, the prosthesis should be implanted using a kinematic or anatomic alignment. The anterior femoral cut should be made perpendicular to the sagittal axis of the joint. If the trochlear sulcus is still detectable, drawing Whiteside's line is helpful to identify the sagittal axis. The anterior cut should be perpendicular to the Whiteside's line and parallel to the transepicondylar axis (Fig. 20.4). Its depth should consider that the thickness of the femoral implant should replace the amount of bone and cartilage removed plus any cartilaginous wear.

In patients with PFOA secondary to trochlear dysplasia, PFJ biomechanics is completely distorted. The TL is neutral or externally rotated; the lateral trochlear facet is hypoplastic; the LTI
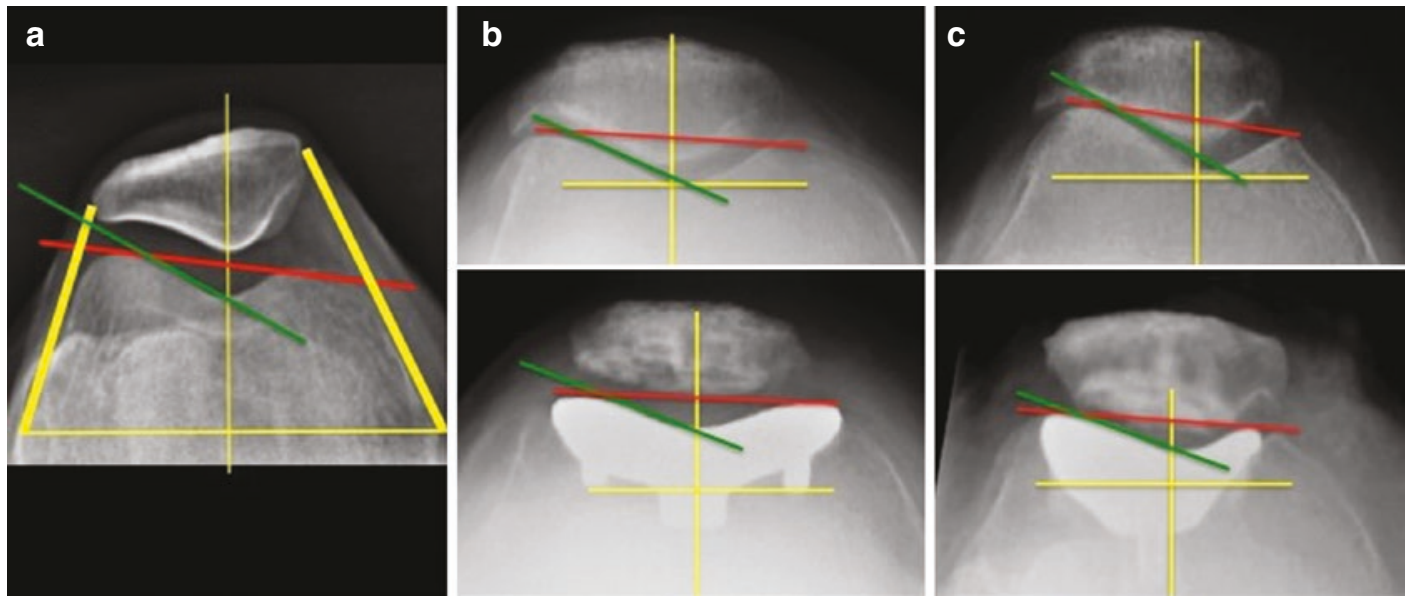

Fig. 20.4 (a) Anatomy of a normal PFJ. The lateral ridge is more prominent than the medial one, consequently the trochlear line (TL, red line) is internally rotated, and the lateral trochlear inclination (LTI, green line) is pronounced. In this condition, the PF ligaments are balanced (yellow lines). (b) Primary PFOA without trochlear dys- plasia. The patella is centred. The PFJ was replaced with an onlay PFA. (c) Primary PFOA without trochlear dysplasia. In this case, an inlay PFA was used. In both cases, the shape and orientation of the joint were left unchanged, and the PFA has an anatomical alignment 
is inadequate; the lateral patellar osteophytes are prominent; the lateral patello-femoral ligament is tensed, and the patella is displaced laterally with a load concentration on the lateral side. PFA should correct all these abnormalities, and it could be made only using an onlay design. The aim of the PFA implant is to correct the cartilage loss and deformity without stressing soft tissue structures, because PFOA is strongly related to soft tissue unbalance. For these reasons, the PFA should be performed with an anterior cut allowing a hypocorrection of the deformity, maintaining patient's morphotype. The lateral facet height must be recreated undercutting the lateral aspect of the trochlea. Anyway, in case of high-grade trochlear dysplasia, the anterior cut should maintain a slight external rotation to accommodate for the abnormally tight lateral retinaculum and abnormally lax medial retinaculum. This adjusted kinematic alignment will obtain a realignment with no or minimal lateral release and minimum risk of overstuffing. Kinematic alignment undercorrects the external rotation of the TL but improves the sulcus angle (SA) and the LTI. The SA depends on the prosthetic shape; the LTI depends on the shape and the rotation of the implant (Fig. 20.5). In any case, internal rotation of the trochlear component relative to the posterior condylar line should be avoided.

After the anterior cut is made, a dedicated milling guide, specific for each design of PFA, creates the allocation for the prosthetic trochlea. The correct size and placement of the trial should be chosen: the distal aspect of the implant should be flush with the articular cartilage both medially and laterally, and its mediolateral width should cover the entire trochlea, with no overstuffing. A high-velocity cutter removes a minimal amount of bone and creates the bed for the prosthesis.
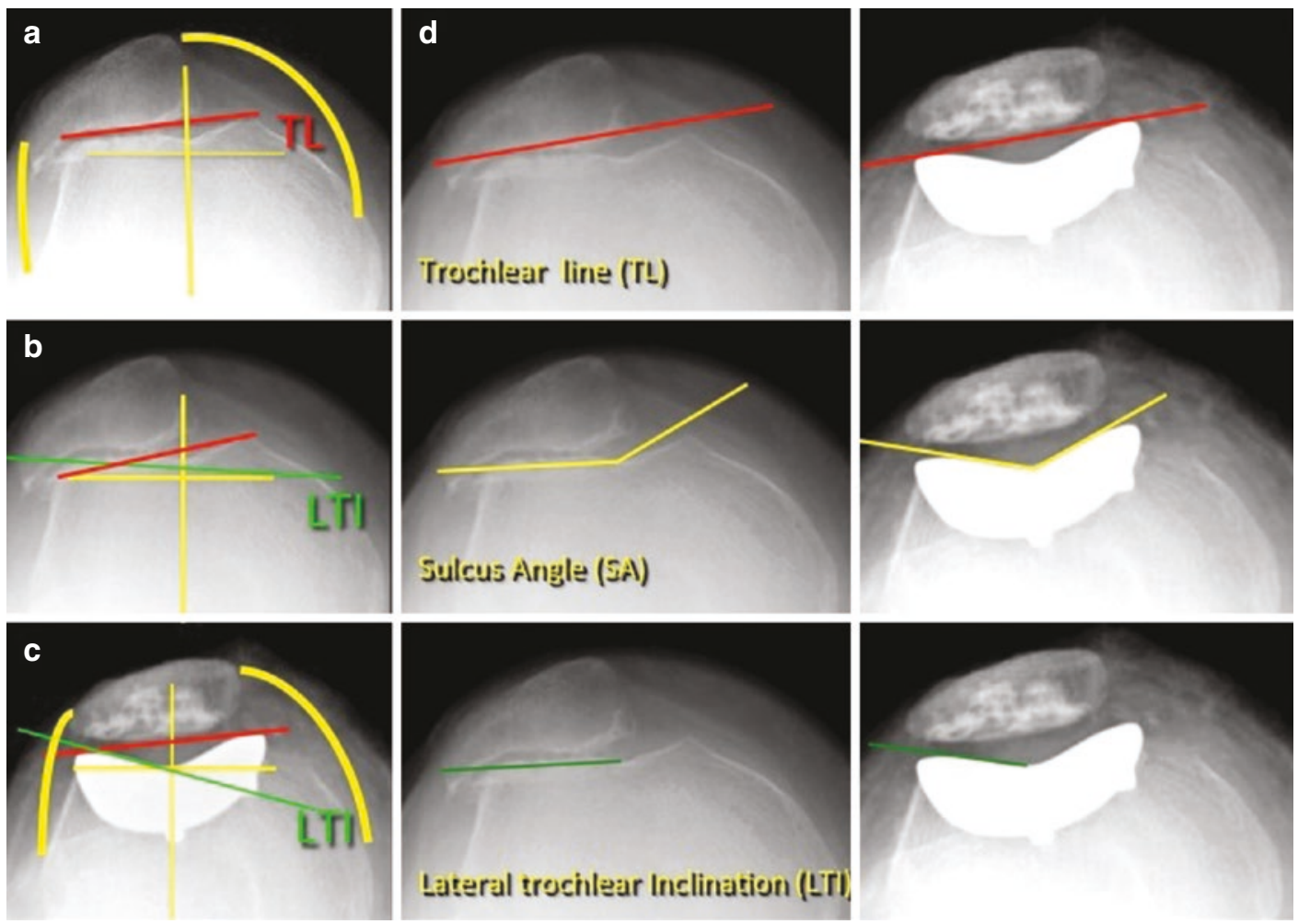

Fig. 20.5 PFOA with a dysplastic trochlea. (a) The lateral aspect of the trochlea is hypoplastic, and consequently the trochlear line (TL, red line) is extrarotated. The medial PF ligament is lax, and the lateral one is tight (yellow lines). (b) The lateral trochlear inclination (LTI, green line) is minimal. (c, d) PFA with an onlay PFA. The TL was partially corrected but remained extrarotated, the sulcus angle improved thanks to the prosthetic design, and the LTI was adequately restored. PF ligaments had only a minimal modification. PFA has a kinematic alignment 
Accurate preparation of the width and depth of the bone bed is crucial to avoid any step in the cartilage-prosthesis transition zone, which could create patellar impingement and clunks.

With the knee in full extension, the patella is resurfaced. As PFOA should be considered pathology of the whole PFJ, we suggest resurfacing the patella recreating the native patellar thickness.

With the trial component in situ and after temporary closure of the capsule with 2-3 stitches or Backhaus clamps, patellar tracking should be checked. The patella should be centred into the trochlea during the whole ROM, without any impingement, clunking or subluxation. In addition, the trochlear and patellar implants should not overhang in order to prevent any soft tissue impingement and pain. When the desirable dimension of any component falls between two different sizes, we suggest choosing the smaller one. Cementing procedure starts from the trochlea and then continues with the patella. After that, PF tracking should be checked again. In PFA, capsular suture is of paramount importance, and, in particular when a lateral approach is performed, it can be modulated to adjust any minimal imperfection of the patellar tracking.

Progressive weight bearing starts the day of surgery as well as both passive and active mobilization. Patients are typically discharged from the Orthopaedic Department on day 2 post-op, after demonstrating the ability to full weight bearing with two crutches and are able to flex the knee at least $90^{\circ}$. In selected cases, outpatient surgery could be performed with a strict follow-up. Case Presentation figure shows an example of a bilateral PFA in a subject with pre-operative subluxated alta patellas.

\subsection{Clinical Evidence of PF Replacement}

Second-generation PFAs have lower revision rates and higher functional outcomes compared to first-generation inlay design prostheses [1, 14-16, 18, 19].

Recent studies reported a survivorship of $91.7 \%$ at 5 years, $83.3 \%$ at 10 years, $74.9 \%$ at
15 years and $66.6 \%$ at 20 years [1]. However, the longer follow-ups were affected by the limited results of the first-generation prostheses. When comparing more recent studies with studies published before 2010, the more recent ones reported a lower annual revision rate (1.93 vs. 2.33). The same study reported that the percentage of patients reporting good or excellent knee function varied between $86.8 \%$ and $92.5 \%$ at 5 years of follow-up. Moreover, reports from high-volume centres reported better outcomes and higher survivorship compared to data extrapolated from registries [1].

We have published our experience of 105 gender-specific PFAs at a mean follow-up of 5.5 years [12]. Sixty-four were isolated PFA, and 41 were UKA + PFA. Both groups showed a clear improvement in ROM, pain, Knee Society Score and UCLA activity score compared to preoperative values. Survivorship of these 105 implants was $95.2 \%$. Consequently, modern PFA could be affordable both isolated than combined with a UKA, leading to excellent functional and survivorship results.

Progression of OA in the tibiofemoral components is the major long-term failure cause in PFA [1]. Dahm et al. demonstrated that patients with PFOA secondary to trochlear dysplasia had significantly less radiographic evidence of tibiofemoral joint osteoarthritis progression compared with those without trochlear dysplasia at 4 years mean follow-up [19].

\section{Case Presentation}

Fifty-five-year-old female with isolated bilateral PFOA. Preoperative weight-bearing X-rays showed pristine tibiofemoral joints and endstage PFOA secondary to trochlear dysplasia and patella alta.

The patient underwent simultaneous bilateral PFA with an adjusted kinematic alignment technique. The prosthetic trochlea compensated the hypoplasia of lateral condyle and created an adequate sulcus angle; the trochlear line was corrected but remained externally rotated. The highriding patella faced anyway the prosthetic trochlea without the need of further surgical procedures. 

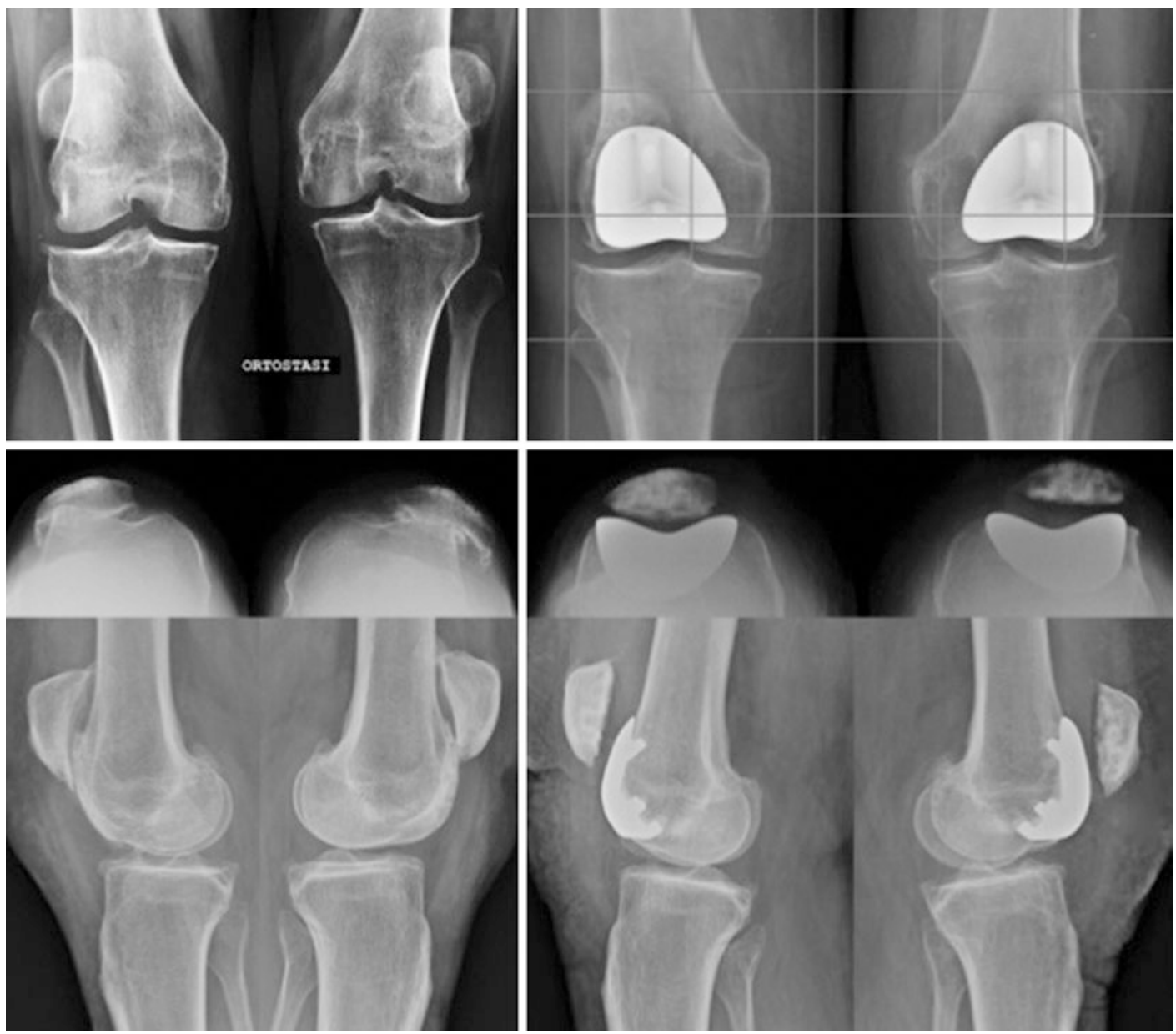

\section{References}

1. van der List JP, Chawla H, Zuiderbaan HA, Pearle AD. Survivorship and functional outcomes of patellofemoral arthroplasty: a systematic review. Knee Surg Sports Traumatol Arthrosc. 2017;25(8):2622-31. https://doi.org/10.1007/s00167-015-3878-z.

2. Scindler O, Scott N. Basic kinematics and biomechanics of the PFJ. Acta Orthop Belg. 2011;77:421-31.

3. Bandi W. Chondromalacia patellae and arthritis of the patellofemoral joint. Helv Chir Acta. 1972;11:1-70.

4. Walker PS. Contact areas and load transmission in the knee. In: American Academy of Orthopedic Surgeons: symposium on reconstructive surgery of the knee. Saint Louis: Mosby Company; 1978. p. 26-36.

5. Romagnoli S, Verde F, Zacchetti S. Bicompartmental prosthesis. In: Confalonieri N, Romagnoli S, editors. Small implants in knee reconstructions. Milan: Springer; 2013. p. 105-16.
6. Csintalan RP, Schulz MM, Woo J, McMahon PJ, Lee TQ. Gender differences in patellofemoral joint biomechanics. Clin Orthop. 2002;402:260-9.

7. Mahfouz M, Booth R Jr, Argenson J, Merkl BC, Abdel Fatah EE, Kuhn MJ. Analysis of variation of adult femora using sex -specific statistical atlases. Presented at Computer Methods in Biomechanics and Biomedical Engineering Conference; 2006.

8. Varadarajan KM, Gill TJ, Freiberg AA, Rubash HE, Li G. Gender differences in trochlear groove orientation and rotational kinematics of human knees. J Orthop Res. 2009;27:871e8. https://doi.org/10.1002/jor.20844.

9. McAlindon TE, Snow S, Cooper C, Dieppe PA. Radiographic patterns of osteoarthritis of the knee joint in the community. Ann Rheum Dis. 1992;51:844-9.

10. Dejour D, Allain J. Histoire naturelle de l'arthrose fémoro-patellaire isolée. Rev Chir Orthop. 2004;90:1S69-1S129.

11. Guilbert S, Gougeon F, Migaud H. Evolution de l'arthrose fémoro-patellaire isolée: devenir à 9 ans 
de recul moyen de 80 genoux non opérés. Rev Chir Orthop. 2004;90:1S69-86.

12. Romagnoli S, Marullo M. Mid-term clinical, functional, and radiographic outcomes of 105 genderspecific patellofemoral arthroplasties, with or without the Association of Medial Unicompartmental Knee Arthroplasty. J Arthroplast. 2018;33:688-95.

13. Romagnoli S, Marullo M. What are the limits for unicompartmental knee arthroplasty? In: The young arthritic knee. Abstract book of 16èmes Journées Lyonnaises de Chirurgie du Genou 2014 Bonnin et al Editors Sauramps Medical; 2014.

14. Leadbetter WB, Ragland PS, Mont MA. The appropriate use of patellofemoral arthroplasty: an analysis of reported indications, contraindications, and failures. Clin Orthop Relat Res. 2005;436:91e9.

15. Lonner JH. Patellofemoral arthroplasty: the impact of design on outcomes. Orthop Clin North Am. 2008;39:347e54. https://doi.org/10.1016/j.ocl.2008. 02.002 .
16. Lonner JH, Bloomfield MR. The clinical outcome of patellofemoral arthroplasty. Orthop Clin North Am. 2013;44:271e80. https://doi.org/10.1016/j.ocl. 2013.03.002.

17. Carrillon Y, Abidi H, Dejour D, et al. Patellar instability: assessment on MR images by measuring the lateral trochlear inclination-initial experience. Radiology. 2000;216:582-5.

18. Lustig S, Magnussen RA, Dahm DL, Parker D. Patellofemoral arthroplasty, where are we today? Knee Surg Sports Traumatol Arthrosc. 2012;20:1216e26. https://doi.org/10.1007/s00167012-1948-z.

19. Dahm DL, Kalisvaart MM, Stuart MJ, Slettedahl SW. Patellofemoral arthroplasty: outcomes and factors associated with early progression of tibiofemoral arthritis. Knee Surg Sports Traumatol Arthrosc. 2014;22(10):2554-9. https://doi.org/10.1007/s00167014-3202-3.

Open Access This chapter is licensed under the terms of the Creative Commons Attribution 4.0 International License (http://creativecommons.org/licenses/by/4.0/), which permits use, sharing, adaptation, distribution and reproduction in any medium or format, as long as you give appropriate credit to the original author(s) and the source, provide a link to the Creative Commons license and indicate if changes were made.

The images or other third party material in this chapter are included in the chapter's Creative Commons license, unless indicated otherwise in a credit line to the material. If material is not included in the chapter's Creative Commons license and your intended use is not permitted by statutory regulation or exceeds the permitted use, you will need to obtain permission directly from the copyright holder. 